\title{
EL CARÁCTER PRÁCTICO DE LOS DEBERES JURÍDICOS*
}

\author{
María Cristina Redondo
}

CONICET (Argentina)

\section{Introducción}

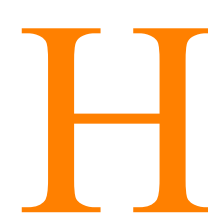

-ay diversas formas en las que un modelo teórico puede ser puesto en tela de juicio. Una de ellas consiste en mostrar que su implementación no permite captar aquello que interesa respecto de un problema, o que lo hace de un modo notoriamente defectuoso. Ésta es una de las críticas que se le formulan al modelo positivista cuando se trata de explicar el carácter práctico del derecho, es decir, su capacidad para determinar la conducta de los ciudadanos y jueces a los que se dirige.

Un reflejo de la distinción positivista entre lo que el derecho es y lo que debe ser es la separación nítida entre los problemas de identificación del derecho, y los problemas relativos al deber de aplicación u obediencia de sus normas. Conforme a algunas propuestas positivistas, el primero es un problema al que la teoría jurídica debe brindar una respuesta; pero el segundo es un problema práctico que no puede ser resuelto por la teoría general del derecho. La respuesta a la cuestión sobre si se debe aplicar u obedecer el derecho depende, contingentemente, de lo que requiera cada sistema jurídico, y de si su contenido está o no en conflicto con alguna consideración moral aplicable a la situación particular. En otras palabras, el derecho no tiene por sí mismo carácter práctico, dicha cualidad depende de la corrección moral de su contenido. Desde esta perspectiva, que exista un derecho no equivale a que exista un deber de hacer lo que él establece, i.e. de obedecerlo o de aplicarlo.

Conforme a algunos de sus críticos, la separación que el positivismo propone entre el problema de identificación del derecho, por un lado, y el

\footnotetext{
${ }^{*}$ Este trabajo ha sido realizado gracias al apoyo del CONICET, Fundación Antorchas, y la Fundación Caja de Madrid.
} 
relativo al deber de aplicación u obediencia, por otro, es viable ${ }^{1}$; pero pone en evidencia que el modelo teórico del positivismo no logra explicar el carácter práctico del derecho. En efecto, desde esta posición se subraya que uno de los objetivos fundamentales de cualquier teoría jurídica consiste en dar una explicación de la normatividad de las disposiciones del derecho, es decir, de su carácter de razón capaz de determinar la acción de los agentes. No captar correctamente este rasgo, o pasarlo por alto, equivale a no explicar por qué el derecho es una institución con relevancia práctica. En este sentido, sea o no definitorio del derecho, lo importante es saber de qué modo y bajo qué condiciones éste establece genuinos deberes de actuar, i.e. razones para hacer lo que nos requiere. La falla del positivismo consiste, o bien en que se desinteresa de la explicación de este rasgo práctico fundamental, o bien en que al hacerlo debe abandonar una de sus premisas básicas, y reconocer que la capacidad práctica del derecho sólo se explica a través de su relación con la moral ${ }^{2}$. Esta última crítica se sostiene en la idea según la cual, sólo las razones morales constituyen «genuinas» razones para la acción, i.e. son las únicas generadoras de un auténtico deber de actuar. Consecuentemente, sólo en virtud del apoyo en razones morales el derecho puede ser normativo y tener carácter practico ${ }^{3}$.

\section{La normatividad del derecho}

La idea de normatividad es notoriamente ambigua. Se suele sostener que lo que confiere carácter normativo a un enunciado es el uso de dicho enunciado con la intención de guiar o dirigir la conducta de otro agente. Por ejemplo, si admitimos que todas las disposiciones de lo que conocemos como el antiguo derecho romano fueron establecidas con esta intención, podemos afirmar que el derecho romano aún hoy es normativo. Bajo este concepto, el predicado «normativo» atribuido a un enunciado significa que ha

\footnotetext{
${ }^{1}$ Conforme a otro tipo de crítica, esta separación no es posible. La imposibilidad se basa en que la pregunta por la identificación del derecho está necesariamente unida a la pregunta acerca de cómo debemos actuar desde un punto de vista moral. Identificar el derecho es un proceso constructivo en el que se proyectan nuestras convicciones acerca de cómo comportarnos correctamente. Cf. Dworkin, Law's Empire, Harvard University Press, Cambridge, 1986, chap. II; también Nino, Carlos S. Derecho, Moral y Política, Ariel, Barcelona. 1994, cap. III.

${ }^{2}$ En esta crítica coinciden, inclusive, algunos autores que mantendrían una teoría positivista para identificar el derecho. Por ejemplo, ella se encuentra en los primeros trabajos de Carlos Nino y, en la literatura española, en numerosos trabajos de Manuel Atienza, Juan Ruiz Manero, Juan Carlos Bayón, etc. Desde esta perspectiva, las normas jurídicas no bastan para justificar la decisión judicial, ya que ellas no constituyen razones operativas.

${ }^{3}$ En opinión de Joseph Raz, algunas de las estrategias que explican la normatividad del derecho basándose en su relación con las razones morales son compatibles con el positivismo. Cf. Raz, Joseph, "Authority, Law and Morality», en Ethics in The Public Domain. Essays in The Morality of Law and Politics, Clarendon Press, Oxford, 1994, pp. 210-237.
} 
sido emitido con el propósito de obligar, prohibir o permitir algo, y es independiente de la efectiva relevancia práctica que tenga el enunciado del que se predica. Esto explica porqué no es ésta la noción de normatividad que interesa en la discusión actual, especialmente preocupada por el problema de la relevancia práctica del derecho.

Por lo general, en la discusión sobre la normatividad del derecho el interés no se dirige a la mera identificación de enunciados formulados con la intención de obligar, prohibir o permitir conductas. Que un enunciado contenga estos términos deónticos, y/o que se use con la intención de guiar conducta, no garantiza que dicho enunciado adquiera relevancia práctica, i.e. dé efectivamente lugar a un deber de aplicación y obediencia por parte de los jueces y ciudadanos. Según esta perspectiva, la teoría del derecho tiene que explicar bajo qué condiciones surgen estos deberes. Es decir, bajo qué condiciones las disposiciones jurídicas logran constituir razones para la acción que requieren. La noción de razón para la acción puede entenderse de distintas maneras, en cualquier caso, conforme a esta segunda perspectiva, el concepto de normatividad está internamente vinculado con dicha noción. En este sentido, es claro que las disposiciones del antiguo derecho romano, en general, hoy carecen de normatividad.

Por último, me interesa destacar que, aun entre quienes vinculan la noción de normatividad con la de razón para la acción, existe una discusión interna que, a menudo se plantea como un interrogante acerca de cuándo un enunciado o consideración es «genuinamente» normativo. Así, se afirma que un enunciado no es «genuinamente» normativo si constituye sólo una razón ordinaria que se suma, como una más, a las restantes razones existentes para hacer o dejar de hacer algo. Conforme a una de las propuestas más sobresalientes en esta discusión, lo que caracteriza a las normas es que ellas constituyen un tipo especial de razón que, a la vez que provee una razón para la acción, es excluyente de otras razones ordinariamente aplicables. En este trabajo no me referiré a esta específica discusión ${ }^{4}$.

${ }^{4}$ Sin embargo, cabe destacar que se trata de un tema fundamental en relación a la discusión en desarrollo. Dado que el derecho regula conductas, primordialmente, a través de reglas es preciso explicar en qué se diferencian las reglas de las restantes razones ordinarias. A su vez, es también preciso enfrentar la acusación de irracionalidad que pesa sobre las acciones y decisiones cuya razón es el seguimiento de una regla. Al respecto existe una extensa bibliografía. Véase, por ejemplo, Raz Joseph, Practical Reason and Norms, (1 ed), Hutchinson \& Sons Ltd., London, 1975. Schauer, Frederick, Playing by the Rules; A Philosophical Examination of Rule-Based Decision-Making in Law and Life, Clarendon Press, 1991. Postema, Gerald, J., «Positivism, I Presume?... Comments on Schauer's 'Rules and the Rule of Law'», Harvard Journal of Law \& Public Policy, Vol. 14, N3, 1991, pp.797-822. Bayón, Juan Carlos, «Sobre la racionalidad de dictar y seguir reglas». Doxa, Alicante, 1996, pp. 143-162. Goldman Alan H., «Rules in The Law», Law and Philosophy, 16, 1997, pp. 581-602. Russ Shafer-Landau, «Moral Rules», Ethics, 107, 1997, pp. 584-611. 
Frente a la objeción señalada en la introducción, el desafío del positivismo consiste en mostrar que las tesis y distinciones que él propone no obstan a la explicación del referido carácter práctico del derecho. En otros términos, dada la admisión unánime de que el sentido de normatividad sobre el que interesa discutir es el vinculado con la capacidad del derecho para determinar la acción constituyendo razones, el positivismo, si acepta los términos de la discusión, debe mostrar que este rasgo puede explicarse sin dificultad dentro de su esquema teórico. Si el positivismo no logra dar cuenta del carácter práctico del derecho con independencia de la moral, debe reconocer que su propuesta de separar estos dos fenómenos normativos le impide captar este aspecto esencial del derecho.

\section{Deberes jurídicos y aplicabilidad}

Un modo aparentemente plausible de identificar deberes jurídicos es el que se apoya sobre el concepto de aplicabilidad. Conforme a este punto de vista, la verdad de los enunciados de deber jurídico depende, no de las normas que pertenecen al sistema de referencia, sino de aquellas que deben aplicarse. Es posible que una norma pertenezca a un sistema jurídico y, sin embargo, no deba ser aplicada. En este sentido, no impone el deber de actuar conforme a ella. A la inversa, puede suceder que una norma no pertenezca al sistema y, sin embargo, los jueces tengan el deber de aplicarla. En este caso, y en relación a ese sistema, es verdadero el enunciado según el cual el destinatario debe hacer lo que la norma dices.

Una reflexión preliminar: Si para analizar el deber impuesto por las normas jurídicas se recurre a la idea de aplicabilidad, se debe advertir que dicha expresión es ambigua y que, a su vez, puede ser usada tanto descriptiva como normativamente. La distinción de diversos sentidos de aplicabilidad ha dado lugar a una amplia discusión, a la que no me referiré aquí, pero de la que se puede extraer como conclusión lo siguiente: la afirmación de que una norma es aplicable hace referencia, al menos, a dos cosas distintas:

1. informa que un caso, o un conjunto de casos, cae bajo el alcance de una norma,

2. informa que una norma debe aplicarse a un caso, o a un conjunto de casos.

${ }^{5}$ Conf. Bulygin, «Time and Validity» en Deontic Logic, Computational Linguistics and Legal Information Systems, Vol. II, 65-81, Martino, A. (ed.), North Holland Publishing Company, Dordrecht, 1982. También, Moreso, José Juan y Navarro Pablo, «Applicability and Effectiveness of Legal Norms», Law and Philosophy, 16, 1997, pp. 201-219. Estos autores distinguen entre aplicabilidad interna y externa. Dos sentidos que, en ciertos aspectos, son similares a los que propongo más adelante, pero que se distinguen de ellos en varias notas importantes. No es oportuno detenerme aquí estas semejanzas y diferencias. 
Adviértase que el primer sentido de aplicabilidad depende del criterio de individualización de normas que se use, y del contenido concreto que se les atribuya. Este concepto de aplicabilidad es inherente a toda norma, porque una norma siempre regula un conjunto de casos individuales a los que, en este primer sentido, es aplicable. En contraste, es discutible si el segundo sentido de aplicabilidad es o no necesario a toda norma. Por ejemplo, desde un punto de vista Kelseniano, las normas que existen son también aplicables en este segundo sentido. Sin embargo, muchos autores han criticado esta concepción de Kelsen calificándola como pseudo-positivista ${ }^{6}$ o positivista ideológica ${ }^{7}$. Más adelante volveré brevemente sobre esta apreciación.

Cabe destacar que la aplicabilidad de una norma, en cualquiera de los dos sentidos, es siempre relativa a un caso ${ }^{8}$. Teniendo esto presente, un juicio positivo de aplicabilidad en el primer sentido equivale a afirmar que el caso cae bajo el alcance de la norma. En cambio, un juicio positivo de aplicabilidad en el segundo sentido, seguramente no equivale, pero aún es discutible que implique que el caso cae bajo el alcance de la norma. Por ejemplo, el deber de aplicar por analogía una norma a un caso no regulado, pero similar al previsto por ella, muestra que una norma podría ser aplicable -en el segundo sentido, i.e. deber aplicarse- en un caso en el que no es aplicable -en el primer sentido, i.e. no incluido dentro de su alcance?

Como se puede observar, el primer sentido de aplicabilidad es sumamente importante, sin embargo, en el presente contexto sólo es relevante el concepto de aplicabilidad que da apoyo al juicio de deber de aplicar una norma. En lo que sigue de este trabajo me referiré sólo a este sentido de aplicabilidad.

\section{Normas aplicables en virtud de otra norma \\ Normas aplicables en virtud de una práctica}

Decir que una norma N1 es aplicable, en sentido descriptivo, equivale a informar que otra norma N2 prescribe que N1 debe aplicarse. Si la aplica-

\footnotetext{
${ }^{6}$ Ross, Alf, El concepto de validez y otros ensayos, trad. de Genaro Carrió y O. Paschero, Bs.As., Centro Editor, 1969, pg. 25.

${ }^{7}$ Bobbio, Norberto, El problema del positivismo jurídico, trad. de Ernesto Garzón, Fontamara, México, 1991.

${ }^{8}$ Cf. Bulygin, Eugenio, op. cit., p. 66. Este autor se refiere sólo a la noción de aplicabilidad entendida como el deber de aplicar una norma.

${ }^{9}$ Estos dos sentidos de aplicabilidad son muy importantes porque en ellos se apoyan dos sentidos correlativos en los que una razón puede ser derrotada. Estos, a su vez, permiten mostrar las diferencias existentes entre una regla y una razón ordinaria. El presente tema está vinculado con los problemas mencionados en la nota 4 y que, por razones de extensión, no trataré en esta oportunidad.
} 
bilidad de una norma N1 depende del deber impuesto por otra norma N2, es preciso que N2 también sea aplicable; de lo contrario ella no impondría ningún deber. Para juzgar si N2 es aplicable debemos consultar si existe otra norma N3 que así lo establezca y que, a su vez, también sea aplicable. Analizado de esta forma, el juicio sobre la aplicabilidad de una norma -al igual que el análisis usual de la validez basada en la legalidad de la creación- supone una cadena que culmina en una norma respecto de la cual ya no existe otra que prescriba su aplicación. En este punto, la aplicabilidad de las normas, o bien supone un regreso al infinito, o bien exige reconocer que las últimas normas, si son aplicables, lo son en un sentido diferente ${ }^{10}$.

Dentro de la teoría positivista se han ofrecido diversas respuestas a esta pregunta por el deber de aplicación de las últimas normas del sistema ${ }^{11}$. Por ejemplo, conforme a Herbert Hart y Joseph Raz, el deber de aplicación de una norma N1 generalmente depende de otra norma N2 que establece el deber de aplicarla, pero puede también no depender de otra norma. Esto último es lo que sucede con las últimas normas de un sistema, las cuales son aplicables sólo si se constituyen en reglas sociales. Entre los requisitos para la existencia de este tipo de reglas se encuentra la presencia de un grupo de individuos que las respetan y prescriben la aplicación de su contenido. En efecto, una de las condiciones para que una pauta tenga el carácter de regla social es que ella sea generalmente obedecida y aceptada por un grupo de agentes. A su vez, uno de los componentes de esta actitud de aceptación es que quienes la tienen prescriben la aplicación de la norma; es decir, sostienen su aplicabilidad en el sentido normativo del término. Cuando esto sucede en torno a ciertas normas, su aplicabilidad no depende de la existencia de otra norma, sino de la práctica y de la aceptación que las sostiene. Conforme a esta explicación, el hecho de que la mayoría de las normas de un sistema jurídico sea aplicable en virtud de otra norma que así lo prescribe se apoya, necesariamente, en que las últimas normas que prescriben aplicación son aplicables en virtud de una práctica. En otras palabras, según esta perspectiva, lo característico de un sistema jurídico es que sus normas deben ser aplicadas sólo si, en última instancia, reposan en

${ }^{10} \mathrm{Al}$ respecto, Eugenio Bulygin reconoce que los últimos criterios de aplicabilidad son en sí mismos aplicables. El problema es identificar en qué consiste esta propiedad que, según Bulygin, es interna a algunas disposiciones del sistema, i.e. a aquellas que contienen los últimos criterios de aplicabilidad.

${ }^{11}$ No consideraré aquí la propuesta kelseniana apoyada en la presuposición de una norma fundante básica. Esta idea ha sido ampliamente discutida y generalmente considerada implausible como explicación positivista del punto de partida de la aplicabilidad de las normas jurídicas. 
una regla social ${ }^{12}$. Es importante señalar cuáles son las alternativas a esta explicación de la aplicabilidad:

a) o bien un regreso al infinito;

b) o bien la subordinación de los deberes jurídicos a los deberes impuestos por otro sistema, que prescriben la aplicación del derecho. Por ejemplo la moral $;{ }^{13}$

c) o bien la admisión de que el derecho en ningún caso logra establecer deber, i.e. carece de relevancia práctica entendida en este sentido.

En conclusión cabe destacar que, por una parte, el predicado descriptivo de aplicabilidad, o deber de aplicación de las normas, no siempre y necesariamente refiere a otra norma; por otra parte, que para predicar aplicabilidad, o deber de aplicación, en sentido informativo es preciso que esta expresión sea previamente empleada en sentido normativo. No podemos afirmar que existe un deber, i.e. que una norma es aplicable, si antes alguien no lo prescribe. Esto significa que el sentido normativo de aplicabilidad es primario respecto al sentido informativo.

\section{La especificidad del derecho como determinante de la acción}

Como es sabido, el positivismo pone el acento en la necesidad de deslindar aquello que el derecho es de aquello que es moralmente correcto, o que es racional hacer, consideradas todas las cosas. Ahora bien, desde algunas perspectivas, esta propuesta de separación se asocia con otra, cuyo carácter necesario para la posición positivista es discutible. Se trata de la separación entre el derecho positivo existente y su fuerza obligatoria. Al respecto, es interesante hacer algunas reflexiones, ya que pueden señalarse argumentos que contradicen esta última distinción y, sin embargo, no ponen en cuestión la primera, i.e. la separación entre lo que el derecho es y lo que es racional, o moralmente correcto hacer.

${ }^{12}$ Conforme a Eugenio Bulygin, el hecho de que los últimos criterios de aplicabilidad del sistema sean aplicables, sin hacer referencia a otra norma, no significa que constituyan una regla social. De acuerdo a lo que estoy sosteniendo aquí, decir que una norma es aplicable, sin que esa aplicabilidad haga referencia a otra norma, presupone que ella es una regla social. Cualquier alternativa a esta explicación está vedada a una posición positivista. Por supuesto, cabe subrayar junto con Eugenio Bulygin que la aplicabilidad de los últimos criterios -en mi reconstrucción: su carácter de regla social- no es una condición necesaria para que el sistema jurídico pueda identificarse como tal, sino para que deba aplicarse, i.e. para que tenga relevancia práctica.

13 Ésta es la posición más generalizada. Conforme a ella, las normas jurídicas son independientes de las normas morales, pero los deberes jurídicos no son independientes de los deberes morales. Es decir, los deberes que pretenden crear las normas jurídicas dependen absolutamente de la conformidad de éstas con la moral. Los aparentes «deberes» jurídicos son deberes entrecomillados ya que sólo la moral puede generar genuinos deberes de acción. 
La separación entre el derecho positivo existente y su fuerza obligatoria usualmente se refleja, entre otras cosas, en la recomendación de no confundir conceptos descriptivos y normativos de existencia y/o validez jurídica. Esto sucede, por ejemplo, cuando se pone énfasis en que una noción descriptiva de validez, como puede ser la basada en la legalidad de la creación de las normas, es siempre independiente de cualquier noción normativa de validez, como lo son la obligatoriedad -deber de seguir las normas por parte de los sujetos normativos- o la aplicabilidad -deber de aplicarlas por parte de los jueces. Asimismo, siempre desde esta perspectiva, se considera un error lógico pasar de un tipo de predicado a otro. Es decir, pasar de la constatación de una propiedad empírica, como es el hecho de que ciertas normas hayan sido legalmente creadas, a la conclusión de que se debe hacer lo que ellas dicen; donde la noción de deber se usa sin aditamentos ni cualificaciones. Lo contrario, se afirma, significaría cometer la falacia naturalista ${ }^{14}$.

Tal como lo han señalado sus críticos, la posición que hace estas distinciones se encuentra con cierta dificultad al momento de explicar la relevancia práctica del derecho, porque según ella la identificación del derecho que es no nos dice nada acerca de lo que debemos hacer. El positivismo, en tanto teoría jurídica, da herramientas sólo para identificar el derecho que es; pero nada nos dice acerca de su fuerza obligatoria, i.e. acerca de si existe un deber. Si un positivista extrajese alguna conclusión respecto a lo que se debe hacer sería acusado de violar la premisa definitoria del positivismo: la distinción ser-deber ser, y de caer en lo que se ha denominado positivismo ideológico o pseudo positivismo ${ }^{15}$.

Ahora bien, la reflexión que quiero presentar consiste en señalar que esta separación entre ser y deber ser sin cualificaciones -y las distinciones conceptuales con las que ella se compromete- no se sigue de, ni está asociada necesariamente a la distinción entre lo que el derecho es y aquello que es racional, o moralmente correcto hacer. Al menos, no se sigue antes de agregar una premisa adicional que el positivismo en modo alguno está requerido a aceptar. Me refiero a la formulación usual del denominado principio de unidad del razonamiento práctico. Este principio, en la interpretación que lo hace relevante en este contexto, sostiene que hay un único concepto de deber, y que aquello que alguien debe hacer no es algo que admita cualificaciones, i.e. algún tipo de relativización. Existe un conjunto de principios prácticos que constituyen deberes «genuinos». Dichos principios, que son los de mayor jerarquía en el razonamiento práctico, son prin-

${ }^{14}$ La idea de falacia naturalista se toma de G. E. Moore, Principia Ética, Oxford University Press, Oxford, 1975, p. 10.

${ }^{15}$ Ésta es la crítica que, como mencioné antes, se le formula a Kelsen. 
cipios morales que dan homogeneidad y prevalecen sobre los restantes discursos prácticos. Estos últimos lograrán establecer «genuinos» deberes sólo si están de acuerdo con los primeros, o si regulan ámbitos indiferentes desde la perspectiva de aquéllos ${ }^{16}$.

Ciertamente, si se asume esta premisa, por coherencia, debe también asumirse que no se puede pasar de lo que el derecho es a conclusiones de deber. Sin embargo, el positivismo no tiene por qué adoptar como premisa el principio de unidad del razonamiento práctico, así entendido. Por el contrario, una opción disponible es afirmar que el derecho constituye genuinos deberes jurídicos; es decir, aquello que el derecho es significa la existencia de un auténtico deber, i.e. la existencia de razones justificatorias. Estos deberes tienen la peculiaridad de que se identifican, siempre, por su relación con ciertas fuentes sociales; aunque ello no implica que las normas que los imponen sean reducibles a dichas fuentes sociales ${ }^{17}$. Asimismo, estos deberes no constituyen ninguna razón justificativa de carácter moral, ni menos aún un balance general acerca de lo que es racional hacer. Del deber jurídico de actuar no se puede extraer ninguna conclusión acerca del deber moral o racional de actuar, no porque el deber jurídico sea un deber subordinado o debilitado respecto al deber moral, sino porque es un deber de otro tipo, independiente de cualquier deber moral o de racionalidad. A su vez, esto no significa que el deber jurídico carezca necesariamente de valor moral, o sea irracional. Lo único que significa es que estos rasgos, i.e. la conformidad con un deber moral o racional de actuar, no forman parte de su significado y son cualidades contingentes, que puede o no tener.

${ }^{16}$ Cf. Nino, Carlos S., La validez del derecho, Astrea, Bs.As. 1985, pp. 125-137. También, «Breve nota sulla struttura del ragionamento giuridico», Ragion Pratica, Milano, 1993, pp. 32-37.

${ }^{17}$ Creo que esta aclaración es imprescindible porque en algunos contextos se critica al positivismo sobre la base de un error. La tesis de las fuentes sociales del derecho en general, de las normas y/o los deberes jurídicos, en particular, no convierte al derecho, a las normas o a los deberes, en hechos. Estos últimos, dentro de una teoría positivista (no realista) son entidades abstractas que la teoría está dispuesta a admitir que existen o son válidas dentro de un específico sistema jurídico, sólo si se dan ciertas condiciones. Entre las condiciones que una teoría positivista defiende está la producción de ciertos hechos sociales. Respecto a la crítica del positivismo basada en este argumento véase Nino, Carlos S., La validez del derecho, Astrea, Bs.As., 1985, pp. 134-5. Asimismo, Derecho, moral y política, Una revisión de la teoría general del derecho, Ariel, Barcelona, 1994, pp. 23-4. También, Introducción al Análisis del derecho, Astrea, Bs.As. 1984, pp.78-81. Véase también, Peczenik, Aleksander y Urbina, Sebastian, 'Why Officials? On Legal Positivism Old and New', Rechtstheorie 27, 1995, p. 152. Un argumento más desarrollado en relación a este tema puede encontrarse en Redondo, M. Cristina, La noción de razón para la acción en el análisis jurídico, Centro de estudios constitucionales, Madrid, 1996, pp. 163-69. 
Adviértase que ésta no es una tesis que ponga en cuestión el denominado principio de Hume, o que cometa algún tipo de falacia ${ }^{18}$. Por el contrario, puede decirse que a partir del rechazo de la existencia de un concepto único de deber, y de la asunción de una definición precisa de lo que es un deber jurídico, saca todas las consecuencias de la idea de Hume, subrayando la necesidad de no confundir propiedades diversas. A partir de un deber jurídico, cuya definición es independiente del deber moral y del balance global de razones, no se puede obtener ninguna conclusión respecto a lo que se debe hacer desde una perspectiva moral, o racional. Esta posición no sostiene que de ciertos hechos sociales se siga lógicamente un deber jurídico. Sí defiende una tesis conceptual y ontológica según la cual:

1. en un caso individual, existe un genuino deber jurídico de actuar cuando a dicho caso es aplicable una norma jurídica que así lo impone, $y$

2. entre las condiciones de aplicabilidad de una norma jurídica no se encuentra la exigencia de ser, ni una razón moral, ni la expresión del balance global de razones.

\section{El principio de unidad y el respeto a todos los puntos de vista}

Lo dicho anteriormente supone el reconocimiento de la ambigüedad de la expresión «deber». En el ámbito jurídico, la noción de deber significa algo diferente que en el ámbito de la moral, o de la racionalidad. Ahora bien, teniendo presente el concepto de deber internamente vinculado con el de razón para la acción, cabe advertir que pueden existir diversos tipos de deber. Algo puede ser debido desde un punto de vista moral, desde un punto de vista religioso, o desde un punto de vista jurídico, etc. Esta relativización de los deberes o razones no supone negar la unidad del razonamiento práctico. Todo individuo puede, y si es racional debe, realizar un cotejo todas las razones -de todos los tipos-, aplicables a un caso individual y, a partir de di-

\footnotetext{
${ }^{18} \mathrm{Al}$ respecto, es útil la distinción que hace Frankena entre tres problemas diferentes: 1. el paso de premisas que no contienen términos normativos a una conclusión normativa, 2. la definibilidad de las propiedades normativas en términos de otras propiedades, y 3. la especificidad de las propiedades normativas en tanto un tipo diferente de las propiedades no normativas. Entre estos problemas, el único que involucraría la comisión de un error lógico es 1 , y es esto lo que intentaba denunciar Hume. Lo que Moore critica como comisión de una falacia es el propio intento de definir los términos normativos, especialmente, si se los define en términos no normativos. Pero, tal como muestra Frankena esto no constituye una falacia si no se muestra antes, y por medios independientes, que dichos términos normativos son en efecto indefinibles en general, o indefinibles en términos no normativos en particular. Antes de haber demostrado por qué estos términos normativos tienen estas características no se puede imputar la comisión de una falacia a quien brinda una definición, sea o no ésta reduccionista. Cf. Frankena, W.K. «The Naturalistic Fallacy», en Theory of Ethics (ed. por Philippa Foot), Oxford University Press, Oxford, 1967, pp. 50-63.
} 
cha evaluación, extraer un deber concluyente de actuar. Justamente, ello significa decidir racionalmente. Las pautas de racionalidad no constituyen un ámbito práctico más, que impone un nuevo conjunto de deberes o razones para la acción, a la par de los deberes jurídicos, morales, religiosos, etc. Las pautas de racionalidad son metacriterios que permiten la evaluación global de los requerimientos impuestos desde los distintos ámbitos prácticos. Actuar o decidir racionalmente es actuar o decidir evaluando todas las razones para actuar existentes en un momento dado ${ }^{19}$.

Cabe subrayar que la relativización señalada tampoco cae en el error del positivismo ideológico. No supone ni un paso lógico incorrecto de un tipo de discurso a otro, ni el otorgamiento de un peso prioritario al derecho en relación a los restantes discursos prácticos. Quien obtiene conclusiones normativas de deber, a partir del derecho, no es un positivista ideológico; dado que el derecho es normativo y permite obtener tales conclusiones. Positivista ideológico es quien sostiene que los deberes jurídicos implican el deber moral o racional de actuar, cometiendo una falacia definista; o bien quien defiende que las razones o deberes jurídicos prevalecen sobre otras razones o deberes, autónomas o heterónomas, que se aplican a un agente. En este último caso, un positivista ideológico es quien asume la idea de unidad del razonamiento práctico y otorga al derecho el papel del único generador de deberes «genuinos», que prevalecen sobre todos los demás.

Esta reflexión sirve para llamar la atención sobre un problema de presentación, que si no se advierte puede generar un pseudo problema teórico dentro de la concepción positivista. De acuerdo a como estoy caracterizando a esta posición, ella presupone la posibilidad de separar: 1. la identificación del derecho (perteneciente y/o aplicable), 2. la identificación de los deberes morales, y 3. la evaluación racional acerca de lo que se debe hacer en un caso particular. Muchas veces, el problema de identificación del derecho se equipara a una pregunta acerca de aquello que el derecho es, y el problema de la identificación de los deberes morales se equipara con la pregunta

${ }^{19}$ Conforme a esta posición, la moral, el derecho, la religión, etc. son distintos sistemas normativos de conducta que imponen, desde su punto de vista, deberes de diverso tipo, a tener en cuenta en la evaluación global. Cada uno de estos sistemas tendrá sus propios criterios de resolución de conflictos internos, pero estos criterios no tienen por qué imponerse a la hora de resolver los conflictos que puedan plantearse entre razones de diverso tipo. Por supuesto, esto contradice explícitamente la idea de «imperialismo» de la moral o la de cualquier otro sistema normativo. Por otra parte, los criterios de racionalidad jerarquizan y resuelven posibles conflictos entre distintos tipos de razones aplicables a un caso, i.e. nos permiten obtener una respuesta, consideradas todas las cosas y todos los puntos de vista. En otras palabras, los principios de racionalidad son meta-normas que jerarquizan, no sistemas normativos (como lo son la moral, la religión o el derecho), sino deberes o razones existentes de acuerdo con distintos sistemas normativos. 
acerca del deber, sin cualificaciones. Esta equiparación es engañosa porque sugiere que los deberes son siempre de un sólo tipo: moral. Sin embargo, si se admite que el discurso del deber -y el de las razones- requiere siempre una cualificación -e.g. un deber es, o bien religioso, o bien político, o bien jurídico, etc.- puede admitirse que la pregunta por la identificación del derecho, en una visión positivista, conduzca a respuestas genuinamente normativas de deber y que, a pesar de ello, no responden -ni pretenden responder- la pregunta acerca de qué debemos hacer desde un punto de vista moral y/o racional. Esta última posición invita a distinguir varios problemas:

En primer lugar, se admite que existen distintos tipos de deber -y de razones para la acción- en virtud del diferente fundamento o raíz de su carácter práctico. Estos diversos fundamentos dan lugar a distintos ámbitos o puntos de vista prácticos. Por ejemplo, existen deberes jurídicos, que generan razones para la acción apoyándose en la coacción y en la aceptación prioritariamente institucional; y a la par, existen deberes morales, que generan razones para la acción y determinan el comportamiento en virtud de la corrección de su contenido. Esto supone que, dentro de lo que el derecho es, corresponde discernir lo que es derecho válido o existente -porque satisface, por ejemplo, el test de la legalidad de su creación- de lo que se debe hacer desde un punto de vista jurídico o, lo que es lo mismo, de lo que es derecho válido en el sentido de jurídicamente vinculante. Sólo estos últimos tienen carácter práctico específico ${ }^{20}$.

En segundo lugar, la pregunta acerca de cuál es el curso racional de acción, es decir, aquello que se debe hacer una vez consideradas todas las cosas, y todos los puntos de vista, exige criterios de resolución de los posibles conflictos entre deberes establecidos en los diversos ámbitos. Si se ha de reconocer auténtico carácter práctico a los diversos ámbitos, estos criterios últimos de jerarquización deben necesariamente satisfacer la condición de ser neutrales respecto de todos ellos, en un doble sentido:

1. Por una parte, no deben ellos mismos pertenecer a ninguno de los ámbitos prácticos que están en conflicto, y entre los cuales pretenden mediar. Esto es lo que no respetan quienes afirman que estos criterios, por el hecho de ser últimos, son morales.

${ }^{20}$ Obviamente, no hay ningún motivo para no llamarles «deberes» o «normas» también a los primeros, i.e. al derecho válido o existente. En este caso, hay que subrayar la ambigüedad de «norma» y «deber». Donde en un caso se hace referencia al concepto hilético de norma y al deber en sentido externalista, y en otro se hace referencia a la normatividad en el sentido de carácter práctico y al deber en sentido internalista, que implica una razón para la acción. En este contexto le estoy llamando «deber» sólo a aquello que está dotado de carácter práctico, y reservo la calificación de derecho existente o válido para las normas que pertenecen a un sistema, sin considerar si generan o no razones para la acción. 
2. Por otra parte, deben jerarquizar razones o deberes, y no sistemas normativos. Es decir, no deben dar prioridad necesaria a ninguno de los ámbitos prácticos. Esto es lo que no respetan quienes sostienen que la moral prevalece ante cualquier otro discurso práctico ${ }^{21}$.

Los criterios de resolución de conflictos pueden denominarse «de racionalidad» en la medida en que permiten obtener una conclusión de deber consideradas todas las cosas, y respetando auténticamente los distintos tipos de razones existentes. Es importante subrayar que lo que normalmente se presenta como una posición que fragmenta el razonamiento en diversos ámbitos y tipos de razones, bajo esta descripción se muestra como una posición enteramente compatible con la unidad del razonamiento, sólo que, a diferencia de como es generalmente entendida, esta idea de unidad respeta todos los puntos de vista sin dar prioridad necesaria o absoluta a ninguno.

\section{Consideraciones finales}

A modo de conclusión, puede decirse que hay dos formas de presentar la posición positivista respecto al carácter práctico de las normas jurídicas, i.e. respecto de su posible calidad de razones para la acción. Estas formas dependen de si se presupone o no un único concepto de deber, y de si se presupone o no que los deberes que implican razones son de un sólo tipo. En otros términos, depende de si se presupone un principio de unidad del razonamiento práctico que por estipulación conceptual da prioridad a la moral, o si, por el contrario, no se estipula por anticipado a qué ámbito práctico corresponde la prioridad en cada caso.

Ahora bien, quienes aceptan la presentación usual del principio de unidad del razonamiento práctico entienden que el deber de actuar -sin cualificaciones- depende de la corrección moral, es decir de la consideración de todas las razones aplicables a un caso, desde un punto de vista moral. El principio de unidad, así entendido, es la premisa que coherentemente conduce a la conclusión según la cual el deber de hacer lo que las normas jurídicas prescriben, i.e. su capacidad para determinar la acción, depende necesariamente de la moral. Las normas jurídicas no tienen en sí mismas capacidad para determinar conductas, i.e. fuerza vinculante o normatividad propias.

En contraste, si se acepta que el discurso del deber es ambiguo, y que además hay diversos ámbitos prácticos que pueden, por sí mismos, ser relevantes en un balance unitario y global, se presupone que estos ámbitos prácticos pueden generar distintos tipos de auténticos deberes o razones que participan en el balance. Ninguna de estas razones es, por sí misma, abso-

\footnotetext{
${ }^{21}$ Por ejemplo, Garzón Valdés, Ernesto, «Algo más sobre la relación entre derecho y moral», Doxa, N8, Alicante, 1990. pp. 119-130.
} 
luta o concluyente. Qué razón prevalezca en una ocasión individual, dependerá de un cálculo racional que evalúe todos los tipos de razones conforme a criterios que satisfagan la condición de neutralidad antes señalada. Así, el deber jurídico, i.e. la fuerza vinculante de las normas jurídicas, se convierte en un rasgo específicamente jurídico que puede hacerse depender de la satisfacción de criterios de aplicabilidad independientes de los dictados de la moral. Una norma que satisface los criterios jurídicos de aplicabilidad, por ello mismo, impone un deber jurídico de actuar: tiene fuerza obligatoria desde un punto de vista jurídico $y$ es relevante en un balance global de razones. Cabe insistir en que la relevancia de este deber no es de carácter moral. La relevancia de un deber jurídico en tanto tal, i.e. su capacidad de incidir en el momento de responder a la pregunta sobre ¿qué se debe hacer? se basa en que él se encuentra establecido por normas que satisfacen criterios efectivamente apoyados por la presión social.

Cuando desde una perspectiva filosófica se parte de premisas que admiten un principio de unidad del razonamiento práctico y, al mismo tiempo, reconocen que todo sistema jurídico es, por sí mismo, una institución con relevancia práctica; por coherencia, se debe admitir que los requerimientos jurídicos entran, por derecho proprio, en la deliberación acerca de cómo se debe actuar, y se debe garantizar que el principio de unidad del razonamiento respete esta condición. De otro modo, si el principio de unidad consagra la prioridad de la moral, entra en contradicción con la premisa de la que se parte: el derecho no es, por sí mismo, una institución con relevancia práctica. Esto equivale a advertir que la afirmación del principio de unidad que consagra la prioridad de la moral no es compatible con la afirmación de la relevancia práctica del derecho. Quien sostiene el principio de unidad bajo los dictados de la moral, debe ser coherente y admitir la irrelevancia práctica del derecho. Quien quiera admitir la relevancia práctica del derecho, debe ser coherente e impugnar el principio de unidad bajo los dictados de la moral.

Conforme a lo expuesto, el positivismo puede responder al reproche sobre la falta de explicación del carácter práctico del derecho sin tener que recurrir a la moral. Sin embargo, para hacerlo debe admitir la ambigüedad de la noción de deber, la existencia de diversos tipos de razones y un principio de unidad del razonamiento que sea neutral respecto a los distintos sistemas generadores de razones. El hecho de que no se acepten estas premisas explica por qué algunas teorías son claramente positivistas en relación a la identificación del derecho, pero no pueden ofrecer una explicación positivista de su carácter práctico, entendido en términos de razones para la acción ${ }^{22}$.

${ }^{22}$ En esto es claro el ejemplo de Joseph Raz, quien se considera positivista pero, explícitamente, admite que el carácter de razón del derecho sólo puede explicarse por relación a pau- 
Es útil destacar, esquemáticamente, las diferencias que resultan según si se acepta o no la concepción usual del principio de unidad del razonamiento práctico.

Si se rechaza la concepción usual y se acepta un principio de unidad que respete la existencia de distintos tipos de deber, se sigue:

1. Una separación estricta entre el deber jurídico, el deber moral y el deber racional de actuar.

2. Pueden existir genuinos deberes jurídicos de aplicar y obedecer el derecho, independientemente de los dictados de la moral.

3. El carácter práctico del derecho es una nota específicamente jurídica dependiente del apoyo directo o indirecto en la coacción y aceptación.

4. En la medida en que la coacción y la aceptación formen parte del concepto de derecho puede afirmarse que los sistemas jurídicos tienen relevancia práctica, i.e. que generan deberes y constituyen razones capaces de determinar la acción.

Por el contrario, siguiendo la concepción usual que somete todos los discursos prácticos a los dictados de la moral, se sigue:

1. Una separación estricta entre ser y deber -que tiene un único significado moral, y es el único generador de razones.

2. No existen genuinos deberes jurídicos de actuar, independientemente de los dictados de la moral.

3. El carácter práctico del derecho es una cualidad moral, dependiente de la corrección de su contenido.

4. En la medida en que la corrección moral del contenido no forme parte del concepto de derecho no puede afirmarse que los sistemas jurídicos tienen relevancia práctica, i.e. generan deberes que constituyen razones capaces de determinar la acción.

Los argumentos presentados muestran que las teorías positivistas que atribuyen carácter práctico al derecho sin aceptar las premisas mencionadas, relativas al concepto de deber y al principio de unidad del razonamiento:

a) o bien lo hacen sólo en sentido metafórico, sin asumir seriamente lo que esta idea presupone.

b) o bien caen en contradicción con sus propias tesis positivistas, admitiendo que el derecho genera siempre una razón moral para hacer lo que él requiere -que es la posición del positivismo ideológico-;

tas morales. La solución que encuentra este autor es sostener que la separación entre derecho y moral no es una tesis necesaria del positivismo. Sin embargo, autores positivistas como Eugenio Bulygin, Frederick Schauer, José Juan Moreso, Pablo Navarro, Walluchow, etc., que mantienen la tesis de la separación, deberían admitir que no pueden explicar dentro de sus esquemas positivistas el carácter práctico del derecho, entendido como razón para la acción. 
c) o bien dejan de ser positivistas al explicar este carácter práctico. Si el derecho genera una razón para la acción, lo hace gracias a su apoyo en la moral. En cualquiera de estos casos, la posición positivista muestra merecer enteramente la crítica presentada en un comienzo: es incapaz, con sus propias categorías, de dar cuenta del carácter práctico del derecho. 